\title{
Analysis of the Influence of Video-Aware Traffic Management on the Performance of the Dejittering Mechanism^
}

\author{
Kathleen Spaey and Chris Blondia \\ University of Antwerp - IBBT \\ Department of Mathematics and Computer Science \\ PATS Research Group - www.pats.ua.ac.be \\ Middelheimlaan 1, B-2020 Antwerpen, Belgium \\ $\{$ kathleen.spaey, chris.blondia\}@ua.ac.be
}

\begin{abstract}
IPTV over DSL access networks is becoming a reality. This paper studies the impact of the presence of a video-aware traffic management module in the access network on the performance of the video dejittering mechanism at the receiver. The presented numerical results illustrate that with the traffic management module, the packet loss probability at the dejittering buffer decreases. Since the traffic management module will only drop the least important enhancement layer packets of the video in case of congestion, especially the important base layer packets of the video gain from the use of the traffic management module. All results in this paper are obtained using a mathematical model.
\end{abstract}

\section{Introduction}

Today, there are several drivers for the delivery of IPTV (Internet Protocol Television) over DSL (Digital Subscriber Line) access networks. These are both market drivers such as broadband service providers facing ongoing threats from cable operators, as well as technological enablers like improvements in video compression. The delivery of television quality video services over a DSL access network is however more demanding than that of traditional high-speed dataonly Internet services: the video streams are more bandwidth intensive and also their sensitivity to packet loss and jitter (variation in the delay) is greater.

Several access network enhancements for the delivery of video services are possible 1. One of them is video-aware traffic management that during congestion periods selectively discards the information that will least affect the picture quality. More and more, also the endpoints are asked to contribute to a performance increase of the applications. This contribution can take several forms, like the encoding of the information that will be transmitted such that the important information can be separated from the less important information, as in

\footnotetext{
* This work was carried out within the framework of the IWT (Flemish Institute for the promotion of Scientific and Technological Research in the Industry) project CHAMP.
} 
the forthcoming scalable extensions of H.264/AVC (Advanced Video Coding), known as SVC (Scalable Video Coding) 2]. SVC generates a base layer and one or more enhancement layers, where each additional layer contributes to enhancing the video quality. Other examples are letting the sources react to feedback obtained about the status of the network, as in DCCP (Datagram Congestion Control Protocol) [3, absorbing the jitter introduced in the network, etc.

Figure 1 shows the network scenario that will be considered in this paper. Video traffic will be sent in packets from a video server towards $N$ video receivers of a DSL customer via the core network, aggregation network and DSL access network. At the receivers, there is a dejittering buffer before the decoders. Packets that arrive before their playout instant are temporarily stored in this buffer, packets that arrive too late are useless and considered lost. A simultaneous delivery of $N$ video streams to one customer is necessary to allow different televisions of the customer to display different content. The actual number of simultaneous video streams sent to one customer is usually very limited due to bandwidth limitations. Assuming a well engineered core and aggregation network, and considering typical MPEG2 (Motion Pictures Expert Group 2) streams around 3.5 Mbit/s for SDTV (Standard Definition Television), or H.264 HDTV (High Definition Television) streams around $8 \mathrm{Mbit} / \mathrm{s}$, it becomes clear that the bottleneck of the network scenario is the downstream DSL rate. This rate is typically $12 \mathrm{Mbit} / \mathrm{s}$ for ADSL2 (Asymmetric Digital Subscriber Line 2) or $24 \mathrm{Mbit} / \mathrm{s}$ for ADSL2+, but these speeds drop quickly with growing distance from the DSLAM (Digital Subscriber Line Access Multiplexer) [4.

The video traffic is presumed to be compressed in two layers: a base layer and an enhancement layer. The base layer packets are of high importance for the video decoding process at the receiver, while the enhancement layer packets of course contribute to the quality of the video, but can be discarded without error propagation. Further, it is assumed that at the bottleneck point a video-aware traffic management module is present, which during congestion periods prevents the enhancement layer packets to enter the bottleneck buffer in which the traffic of the $N$ video streams is multiplexed. A threshold $T h$ on the content of the bottleneck buffer is used to detect congestion, i.e., while the bottleneck buffer

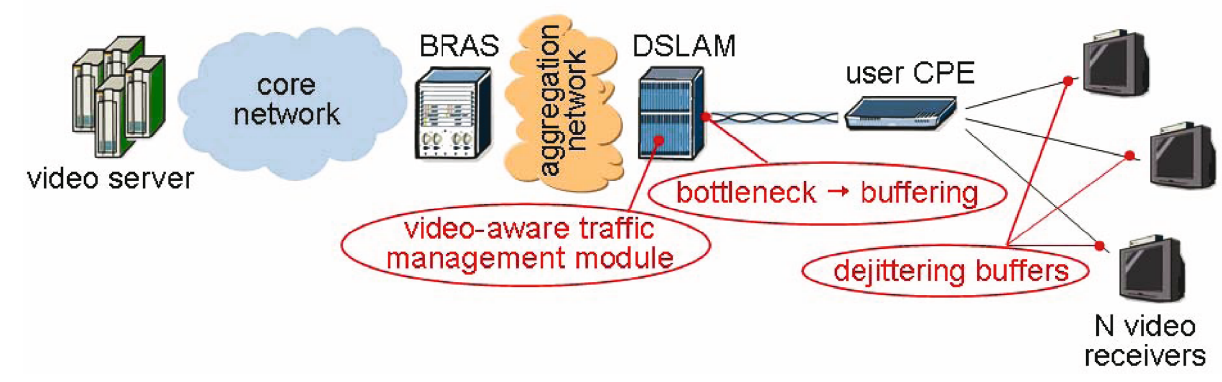

Fig. 1. Network scenario 
content exceeds $T h$, the traffic management module discards all enhancement layer packets.

The aim of this paper is to understand which impact the presence of the videoaware traffic management module in the access network has on the performance of the dejittering mechanism. A mathematical multiplexing model will be used to analyze the discussed scenario. This model and the performance measures that are derived from it are discussed in Section 2 . Section 3 applies the model to numerical examples and discusses the obtained results. The conclusions derived from this work can be found in Section 4.

\section{Model Description and Performance Measures}

The network scenario discussed in Section 1 will be analyzed using a mathematical model. It is assumed that time is divided in units of constant length, called slots, where the length of a slot corresponds to the time needed to place a packet of length $b$ on the bottleneck link of capacity $C$. At the bottleneck, the traffic of the $N$ video streams that is not discarded by the traffic management module, is multiplexed in a finite output buffer of capacity $B$ packets. When this buffer overflows, packets get lost. All packets that were not discarded by the traffic management module and that did not get lost due to buffer overflow at the bottleneck buffer, reach the dejittering buffer of their receiver. But only the packets that reach this buffer on time will also effectively reach the receiver. Packets that arrive after their playout time are useless and considered lost.

\subsection{Video Source Model}

As video source model, consider a two-state Markov modulated Bernouilli source. A typical video contains scenes of different complexity. With constant-quality encoding, more bandwidth is used for complex scenes than for easy scenes. So one state of the Markov source is used to model the easy scenes, the other represents the complex scenes. If $\alpha$, respectively $\beta$, denotes the probability that the source makes a transition to the easy, respectively complex scene state in the next slot, given that it is currently in the complex, respectively easy scene state, then such a source spends a fraction $\pi(c)=\beta /(\alpha+\beta)$ of the time in the complex scene state, and a fraction $\pi(e)=\alpha /(\alpha+\beta)$ in the easy scene state. In both states, the source generates layered video traffic in fixed-sized packets. Such a packet contains either base layer information, or enhancement layer information.

The bit rates at which the packets are generated are denoted by $R_{B+E}(c)$ and $R_{B+E}(e)$, for the complex and easy scene states respectively. The bit rates that correspond to the base layer packets only are symbolized by $R_{B}(c)$ and $R_{B}(e)$. The probability that a source generates a packet in a slot is then $p_{B+E}(c)=$ $R_{B+E}(c) / C$ while the source is in the complex scene state, and $p_{B+E}(e)=$ $R_{B+E}(e) / C$ while the source is in the easy scene state. The probability that a generated packet is a base layer packet is given by $R_{B}(c) / R_{B+E}(c)$ or $R_{B}(e) / R_{B+E}(e)$, depending on the state the source is in when it generates the packet. 


\subsection{Dejittering Principle}

When the video packets traverse the network, they experience a delay that may vary from packet to packet. Consequently, the cadence with which the packets arrive at the receiver's side differs from that with which the video source generated the packets. The task of reducing this jitter is typically performed by placing a dejittering buffer before the decoder. In this buffer, the first packet of the video stream is retained for some time (the dejittering delay) before it is offered to the decoder. From then on, packets are played out with the same cadence as with which the encoder generated the video packet stream. Packets that arrive before their playout instant are temporarily stored in the dejittering buffer, packets that arrive too late are useless and considered lost. For each packet that arrives too late, the dejittering buffer underflows (i.e., runs empty) at the moment such a packet is supposed to be played out.

Choosing the dejittering delay $T_{j i t}$ always involves a trade-off between packets being lost to the video application due to dejittering buffer underflow and their delay. An application must decide about the maximum dejittering delay it can accept, and this in turn determines the fraction of packets that arrive in time to be played out 5 . Namely, if the first packet of a video stream is sent into the network at time 0 , and the packet experiences a delay $\delta_{1}$ before it arrives at the dejittering buffer, then this packet is played out at time $\delta_{1}+T_{j i t}$. If packet $n$ experiences a delay $\delta_{n}$ in the network, and $c_{n}$ denotes the interpacket time at the source between packet 1 and packet $n$, then packet $n$ arrives at the dejittering buffer at time $c_{n}+\delta_{n}$. The target playout time for packet $n$ is then $c_{n}$ time after the first packet, or thus at time $\delta_{1}+T_{j i t}+c_{n}$. So packet $n$ arrives too late at the dejittering buffer if $\delta_{n}>\delta_{1}+T_{j i t}$. This means that the dejittering buffer underflow probability depends on the delay distribution of the packets that arrive at that buffer. Remark however that only the variable component of this distribution matters, which for the considered network scenario is caused by the variable queueing delay at the bottleneck buffer. So if $d^{*}$ denotes the queueing delay experienced by the first packet at the bottleneck buffer, and if $P_{\text {late }}\left(T_{j i t}, d^{*}\right)$ denotes the dejittering buffer underflow probability for a given $T_{j i t}$ and $d^{*}$, then

$$
P_{\text {late }}\left(T_{j i t}, d^{*}\right)=P\left\{D>T_{j i t}+d^{*}\right\},
$$

where $D$ is the random variable that denotes the queueing delay of a packet at the bottleneck buffer. Depending on if $D$ denotes the queueing delay of a random packet, a base layer packet or an enhancement layer packet, (1) gives the probability that respectively a random packet, a base layer packet or an enhancement layer packet arrives too late at the dejittering buffer.

\subsection{Delay Distributions at the Bottleneck Buffer}

From Section 2.2, it became clear that to calculate the dejittering buffer underflow probability for a random packet, a base layer packet or an enhancement layer packet, the probability distributions of the queueing delay experienced at 
the bottleneck buffer by a random packet, a base layer packet or an enhancement layer packet, need to be known. We calculate these distributions using the matrix-analytical approach. In this paper, the details of the calculations are omitted, but a brief and informal outline of the reasoning behind them will be given now.

The bottleneck buffer is modelled as a discrete-time single-server queueing system with a finite capacity of $B$ packets, whose input process is the superposition of the traffic generated by $N$ video sources as defined in Section 2.1, minus the traffic that is discarded at the traffic management module. This queueing system is a level-dependent D-BMAP/D/1/B queueing system where the service times equal one slot. The packets that enter the system are served in FIFO (First In First Out) order. There is a threshold on the content of the system, and depending on whether the content of the system exceeds this threshold or not, a different D-BMAP (Discrete-time Batch Markovian Arrival Process) describes the input process to the queueing system. Namely, a first D-BMAP that describes the superimposed traffic of the $N$ sources consisting of both the base and the enhancement layer packets is used as input process if the system content does not exceed the threshold at the beginning of a slot. In the other case, a second D-BMAP that describes the superimposed traffic consisting of only the base layer packets of the $N$ sources, is used.

If $L(n)$ denotes the number of packets in the system at slot $n$, and $J(n)$ symbolizes the number of sources that are in the complex scene state in that slot, then $\{(L(n), J(n)), n \geq 0\}$ is a two-dimensional Markov chain with state space $\{(l, j) \mid 0 \leq l \leq B, 0 \leq j \leq N\}$ and a $(B+1)(N+1)$-state transition probability matrix $\mathbf{Q}$. First the invariant probability vector of $\mathbf{Q}$, denoted by $\mathbf{x}=\left(\mathbf{x}_{0} \ldots \mathbf{x}_{B}\right)$ with $\mathbf{x}_{i}=\left(x_{i, 0} \ldots x_{i, N}\right)$, is calculated. Element $x_{i, j}$ of vector $\mathbf{x}$ represents the stationary joint probability that in an arbitrary time slot there are $i$ packets in the system and $j$ of the $N$ sources are in the complex scene state.

Once this invariant probability vector is known, the delay distributions at the bottleneck buffer can be calculated. First, a formula for $y_{m}^{(A)}, 0 \leq m \leq B$, which is the probability that a packet that arrives at the bottleneck buffer sees $m$ packets in that buffer upon arrival, is worked out. Remark that since the bottleneck buffer has a finite capacity of $B$ packets, packets that see $B$ packets present in the bottleneck buffer upon their arrival cannot enter that buffer, and will be lost. So $y_{B}^{(A)}$ also equals the packet loss probability of a random packet at the bottleneck buffer. Analogously, also for the probabilities $v_{m}^{(A)}$ and $w_{m}^{(A)}$, $0 \leq m \leq B$, defined as the probabilities that an enhancement layer packet, respectively a base layer packet, sees $m$ packets in the bottleneck buffer upon arrival, an expression is constructed. Again $v_{B}^{(A)}$, respectively $w_{B}^{(A)}$, equals the probability that an enhancement layer packet, respectively base layer packet, gets lost at the bottleneck buffer due to buffer overflow. The probability $d_{m}$ that the queueing delay of a random packet is $m$ slots, is then given by 


$$
d_{m}= \begin{cases}\frac{y_{m}^{(A)}}{1-y_{B}^{(A)}} & \text { if } m \in\{0, \ldots, B-1\} \\ 0 & \text { otherwise }\end{cases}
$$

where the denominator $1-y_{B}^{(A)}$ accounts for the fact that the queueing delay of a packet at the bottleneck buffer only exists for packets that can effectively enter the bottleneck buffer. In a similar way, also the probabilities $d_{m}^{E}$ and $d_{m}^{B}$ that the queueing delay at the bottleneck buffer of an enhancement, respectively a base layer packet, equals $m$ slots, are calculated.

From the invariant probability vector $\mathbf{x}$ of the queueing system, also the fractions of the time $P_{B+E}(c), P_{B}(c), P_{B+E}(e)$ and $P_{B}(e)$ that the video traffic arrives at the bottleneck buffer at the rate $R_{B+E}(c), R_{B}(c), R_{B+E}(e)$ and $R_{B}(e)$ respectively, can be computed.

\subsection{Packet Loss Probabilities}

In Section 2.3, the focus was on the calculation of the delay distributions at the bottleneck buffer, since these distributions determine the underflow probability of the dejittering buffer. However, not all originally generated packets reach the dejittering buffer. In the modeled network scenario, packet loss can occur at three places: at the traffic management module, at the bottleneck buffer, and at the dejittering buffer.

If there is congestion at the bottleneck, the enhancement layer packets of the video streams are already dropped at the traffic management module. The fraction of all packets that are discarded at that point is given by

$$
\frac{P_{B}(c)\left(R_{B+E}(c)-R_{B}(c)\right)+P_{B}(e)\left(R_{B+E}(e)-R_{B}(e)\right)}{\pi(c) R_{B+E}(c)+\pi(e) R_{B+E}(e)},
$$

and the fraction of all enhancement layer packets that are discarded at the traffic management module is then

$$
\frac{P_{B}(c)\left(R_{B+E}(c)-R_{B}(c)\right)+P_{B}(e)\left(R_{B+E}(e)-R_{B}(e)\right)}{\pi(c)\left(R_{B+E}(c)-R_{B}(c)\right)+\pi(e)\left(R_{B+E}(e)-R_{B}(e)\right)} .
$$

Base layer packets are never dropped by the traffic management module.

At the bottleneck buffer, packets will be lost if the bottleneck buffer overflows. The probabilities that a random packet, a base layer packet and an enhancement layer packet respectively, that arrives at the bottleneck buffer cannot enter this buffer, are given by the values $y_{B}^{(A)}, w_{B}^{(A)}$ and $v_{B}^{(A)}$ respectively, which were defined in Section 2.3.

At the dejittering buffer, packets get lost due to dejittering buffer underflow. From (11) it is known that the dejittering buffer underflow probabilities, given $T_{j i t}$ and $d^{*}$, are calculated as

$$
\sum_{m=T_{j i t}+d^{*}+1}^{B-1} d_{m}, \quad \sum_{m=T_{j i t}+d^{*}+1}^{B-1} d_{m}^{B}, \quad \text { and } \sum_{m=T_{j i t}+d^{*}+1}^{B-1} d_{m}^{E},
$$


for a random packet, a base layer packet and an enhancement layer packet respectively.

From all these loss probabilities, the fraction of all generated traffic of a certain type that reaches its receiver can be calculated as the product of the fractions of the traffic of that type that are not lost at the traffic management module, at the bottleneck buffer, and at the dejittering buffer.

\section{Numerical Results and Discussion}

Consider a system with the following characteristics:

- the capacity of the bottleneck DSL link is $C=12 \mathrm{Mbit} / \mathrm{s}$,

- packets have a size of $b=1500$ bytes, so 1 slot corresponds to $1 \mathrm{~ms}$,

- the bottleneck buffer capacity $B$ is 120 packets (or thus $120 \mathrm{~ms}$ ),

- the sources switch on average every 30 seconds to the other scene type,

- the rates at which the sources generate the traffic are $R_{B+E}(c)=3.5 \mathrm{Mbit} / \mathrm{s}$ and $R_{B+E}(e)=2.5 \mathrm{Mbit} / \mathrm{s}$, and

- the rates that correspond to the base layer packets only are $R_{B}(c)=2.4 \mathrm{Mbit} / \mathrm{s}$ and $R_{B}(e)=1.5 \mathrm{Mbit} / \mathrm{s}$.

So one source generates on average $3 \mathrm{Mbit} / \mathrm{s}$. This means that in case the traffic management module is not present, one source puts a load of 0.25 on the bottleneck buffer. A setup with 4 subscribers will be considered.

The Figures 2-4 show for three values of the threshold $T h$ the probabilities that a random packet, a base layer packet and an enhancement layer packet arrive too late at the dejittering buffer, as a function of $T_{j i t}+d^{*}$. Note the different scale of the y-axis in the three figures. A setting of $T h=B=120$ corresponds to the situation where no traffic management module is present at the bottleneck, since irrespective of the bottleneck buffer content, no packets are prevented from entering this buffer. The curves illustrate that for all choices of $T_{j i t}+d^{*}<119$ slots, the underflow probabilities are always smaller when the traffic management module is present than when it is not. The smaller the threshold value that is used, the smaller the underflow probabilities become. Remark from the Figures 3 - 4 that once $T_{j i t}+d^{*}$ becomes larger than the threshold value used, the underflow probability goes down more steeply with increasing $T_{j i t}+d^{*}$ than before. For $T_{j i t}+d^{*}$ equal to or larger than 119 slots, the underflow probability becomes zero in all cases, because a packet that arrives at the dejittering buffer will never have experienced a queueing delay larger than 119 slots in the bottleneck buffer.

Curves as shown in the Figures 2-4 can be used to dimension the parameter $T_{j i t}$ of the dejittering process. This parameter should be set as small as possible to reduce the extra delay introduced by the dejittering process, but large enough to keep the packet loss due to dejittering buffer underflow below some target value. For a certain target underflow probability, the corresponding value for $T_{j i t}+d^{*}$ is read from the curve. Remark that in general the delay $d^{*}$ experienced by the first packet of the video stream is not known at the moment a value for 


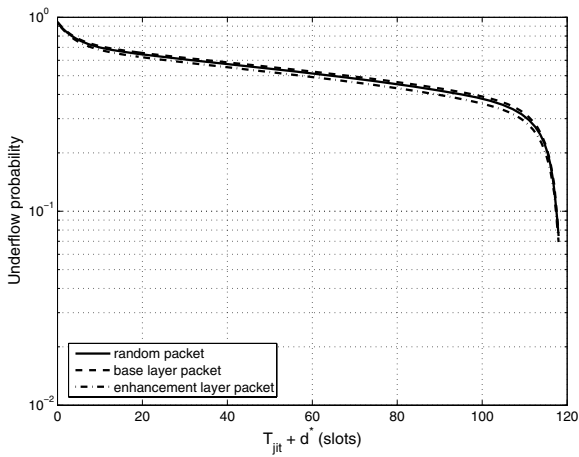

Fig. 2. Underflow probability given $T_{j i t}+d^{*}$, for the scenario with $T h=120$ (no traffic management module)

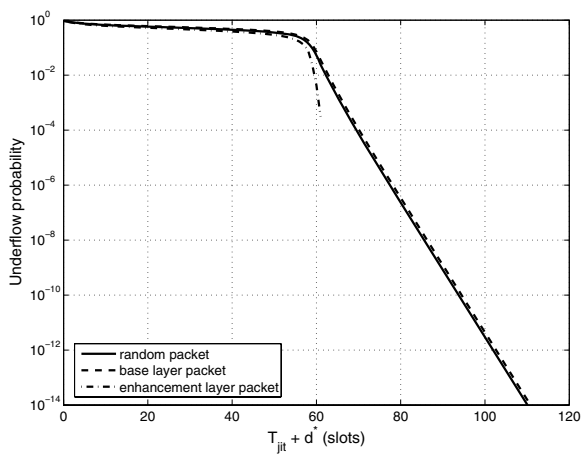

Fig. 4. Underflow probability given $T_{j i t}+d^{*}$, for the scenario with $T h=60$

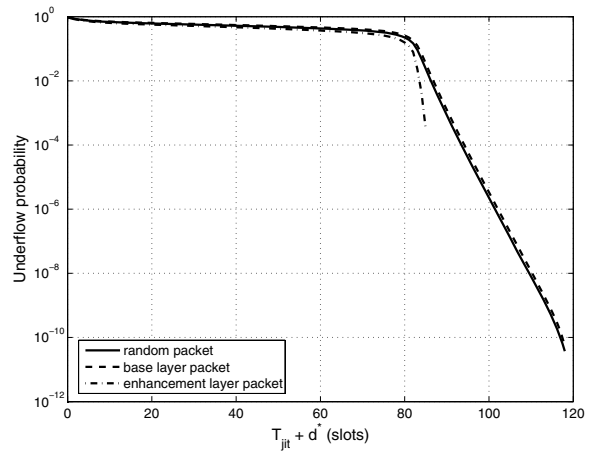

Fig. 3. Underflow probability given $T_{j i t}+d^{*}$, for the scenario with $T h=84$

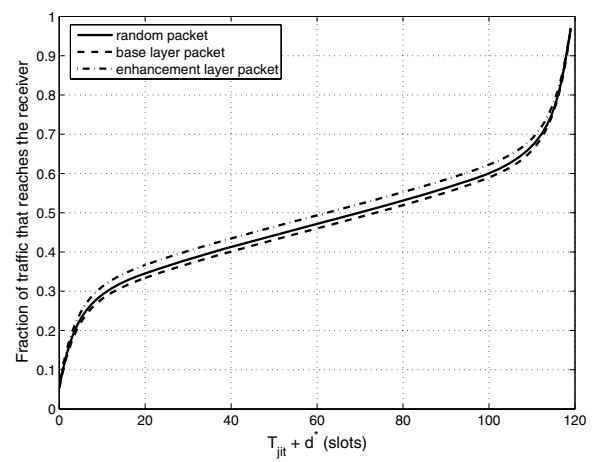

Fig. 5. Fraction of generated packets that reach the receiver. Scenario without traffic management module $(T h=120)$.

$T_{j i t}$ should be set. So under the worst case assumption that the first packet of the video stream experiences a queueing delay of 0 slots in the bottleneck buffer, the worst case value for $T_{j i t}$ is thus found.

From the Figures 2 - 4 it is seen that when the traffic management module is not present $(T h=120)$, there is not much difference between the loss probability due to dejittering buffer underflow for a random packet and for a base layer packet or an enhancement layer packet. When the traffic management module is used, base layer packets have a higher probability than enhancement layer packets to get lost due to dejittering buffer underflow. This is because the underflow probability of the dejittering buffer depends on the delay distribution at the bottleneck buffer, and enhancement layer packets will never experience a delay much larger than the threshold value $T h$ used in the scenario, since at moments the bottleneck buffer content exceeds the threshold, no enhancement layer packets can enter the bottleneck buffer. 
Table 1. Packet loss probabilities at the traffic management module and at the bottleneck buffer

\begin{tabular}{|l|cc|ccc|}
\hline & At traffic management module & \multicolumn{3}{|c|}{ At bottleneck buffer } \\
\hline & random & enhancement & random & base & enhancement \\
\hline$T h=120$ & 0 & 0 & 0.0314 & 0.0325 & 0.0294 \\
$T h=108$ & 0.0316 & 0.0904 & $8.41 \mathrm{e}-06$ & $1.25 \mathrm{e}-05$ & 0 \\
$T h=84$ & 0.0322 & 0.0920 & $9.88 \mathrm{e}-12$ & $1.47 \mathrm{e}-11$ & 0 \\
$T h=72$ & 0.0326 & 0.0931 & $1.15 \mathrm{e}-14$ & $1.72 \mathrm{e}-14$ & 0 \\
$T h=60$ & 0.0331 & 0.0944 & $<1 \mathrm{e}-16$ & $<1 \mathrm{e}-16$ & 0 \\
$T h=48$ & 0.0337 & 0.0963 & $<1 \mathrm{e}-16$ & $<1 \mathrm{e}-16$ & 0 \\
$T h=36$ & 0.0347 & 0.0993 & $<1 \mathrm{e}-16$ & $<1 \mathrm{e}-16$ & 0 \\
$T h=24$ & 0.0368 & 0.1051 & $<1 \mathrm{e}-16$ & $<1 \mathrm{e}-16$ & 0 \\
$T h=12$ & 0.0441 & 0.1259 & $<1 \mathrm{e}-16$ & $<1 \mathrm{e}-16$ & 0 \\
$T h=0$ & 0.35 & 1 & $<1 \mathrm{e}-16$ & $<1 \mathrm{e}-16$ & 0 \\
\hline
\end{tabular}

Table 1 shows for several values of the threshold the packet loss probabilities at the traffic management module and at the bottleneck buffer. Remark that when the traffic management module is not used $(T h=120)$, obviously no packets get lost at it. When it is used, it discards the enhancement layer packets in case of congestion, but never the base layer packets. Therefore, the packet loss probability at the traffic management module of a random packet is $35 \%$ of the packet loss probability of an enhancement layer packet, since from the parameters of the source it is seen that $65 \%$ of all generated packets are base layer packets, and $35 \%$ of the packets are enhancement layer packets. Remark also the extreme case $T h=0$, which corresponds to the scenario where all enhancement layer packets are discarded.

About the packet loss at the bottleneck buffer, it can be read from Table 1 that when the traffic management module is used, only base layer packets get lost due to bottleneck buffer overflow. This is because the bottleneck buffer will only overflow in case of congestion, and during congestion periods the enhancement layer packets are already dropped at the traffic management module (only in examples where the bottleneck buffer space behind the threshold is smaller than the number of sources, it might happen that also enhancement layer packets get lost at the bottleneck buffer). For the scenario with $T h=120$, both types of packets are lost, and it is noticed that much more base layer packets get lost now than when the traffic management module is used.

Based on the values in Table 1, the amount of traffic (random packets, base layer packets or enhancement layer packets) that arrives at the dejittering buffer can be calculated. Namely, this are all packets that were not discarded at the traffic management module and that did not get lost due to buffer overflow at the bottleneck buffer. From these numbers it is then seen that with the traffic management module, all but a very small amount (corresponding to the packet loss at the bottleneck buffer) of the base layer packets reach the dejittering buffer. The smaller the threshold used in the scenario, the more base layer packets reach the dejittering buffer. When the traffic management module is not used, in total 


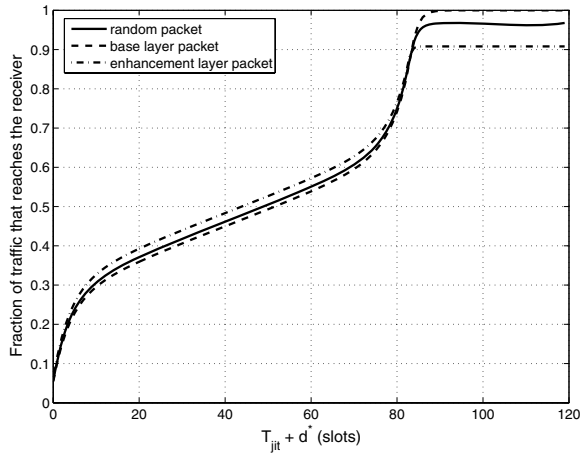

Fig. 6. Fraction of generated packets that reach the receiver. Scenario with $T h=84$.

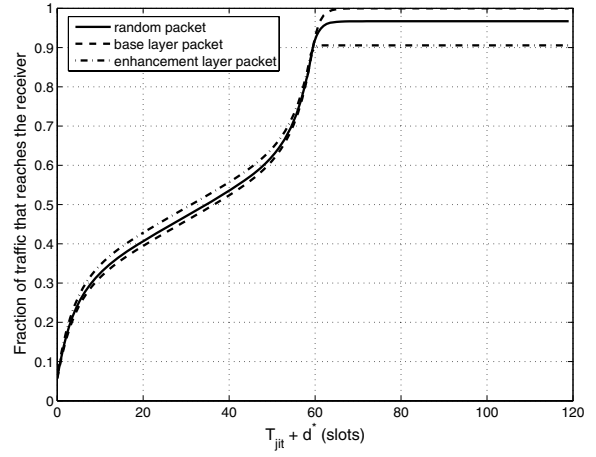

Fig. 7. Fraction of generated packets that reach the receiver. Scenario with $T h=60$.

more packets reach the dejittering buffer, but considerably less of the important base layer packets do.

In case the delay budget is large enough to allow $T_{j i t}+d^{*} \geq 119$ slots, all traffic that reaches the dejittering buffer will also reach the receiver, since the dejittering buffer never underflows. If $T_{j i t}+d^{*}<119$ slots, part of the packets that arrive at the dejittering buffer will get lost at that buffer, because they arrive there after their target playout time. The Figures $5-7$ show for the three examples for which the dejittering buffer underflow probability was shown before, the fractions of the generated traffic that reach the receiver. It is seen that for $T_{j i t}+d^{*}<119$ slots, more video packets (base plus enhancement layer packets) reach the receiver when the traffic management module is used compared to when it is not used. The reason is that in case of congestion, the traffic management module already discards packets, so less traffic is sent into the bottleneck buffer at critical moments. This has as effect that the packet loss at the bottleneck buffer is reduced, and also that the delay the packets experience at the bottleneck decreases, which has a positive effect on the dejittering buffer underflow probability. Further, the figures show that in spite of the fact that base layer packets have a higher probability than enhancement layer packets to get lost at the bottleneck buffer and at the dejittering buffer, end-to-end the base layer packets reach the receiver with a higher probability than the enhancement layer packets when $T_{j i t}+d^{*}$ is larger than the threshold value used in the scenario. The explanation for this is that when $T_{j i t}+d^{*}$ is larger than the threshold, the underflow probability of the base layer packets at the dejittering buffer becomes small compared to the loss probability of an enhancement layer packet at the traffic management module, the latter which is independent of $T_{j i t}+d^{*}$.

So if the goal is to let as much base layer packets as possible reach the receiver, then putting $T h=0$ is obviously the best choice. The lower the threshold however, the more enhancement layer packets are discarded at the traffic management module, and the magnitude of this packet loss increases faster when the threshold position becomes smaller, as can be read from Table 1 And although the enhancement layer packets are the least important packets, they still have 
some value when they reach the receiver, and most likely reducing the base layer packet loss further from for example 1e-9 to much smaller is not worth anymore the loss of several percents of enhancement layer packets. A balance needs thus to be found between where to put the threshold such that at critical moments the bottleneck buffer is discharged because temporarily no enhancement layer packets are sent into it, and the superfluous dropping of the enhancement layer packets.

As becomes clear from the presented results, the choice of the position of the threshold $T h$ in the bottleneck buffer and of the dejittering delay $T_{j i t}$ at the dejittering buffer cannot be uncoupled. Once one of them is fixed, there are limitations on how to choose the other. More concrete, the threshold $T h$ should always be chosen low enough such that the packet loss of the base layer packets at the bottleneck buffer is below a required target, but preferable somewhere in the higher part of the buffer because otherwise there might be a large part of the enhancement layer packets that will never reach their receiver simply because they could not pass the traffic management module. If it is not possible to find a setting for $T h$ such that both requirements are fulfilled, then typically too many connections are accepted into the network. Once the threshold position is chosen, the dejittering delay $T_{j i t}$ should be determined such that $T_{j i t}+d^{*}$ is larger than $T h$, because then the packet loss due to dejittering buffer underflow lies in the region where the underflow probability goes down more steeply with increasing $T_{j i t}+d^{*}$. As mentioned earlier, if the queueing delay $d^{*}$ experienced at the bottleneck buffer by the first packet of the video stream is not known at the moment a value for $T_{j i t}$ should be set, it is best to start from the worst case assumption that the first packet of the video stream will experience a queueing delay of 0 slots.

\section{Conclusions and Future Work}

In this paper, the influence of the presence of a video-aware traffic management module in the access network on the performance of the dejittering mechanism, was studied. All results were derived by applying a mathematical multiplexing model to layered video sources, whose traffic first had to pass a video-aware traffic management module that discards the enhancement layer packets in case of congestion. A threshold Th on the content of the bottleneck buffer is used to detect the congestion.

From the numerical examples, it was concluded that with the presence of the traffic management module, both the packet loss probability at the bottleneck buffer and the dejittering buffer underflow probability (which depends on the delay distribution at the bottleneck buffer) decreases. The reason is that with the traffic management module, part of the packets are already discarded before they enter the bottleneck buffer, so less traffic is sent into the bottleneck buffer at critical moments. Since the traffic management module discards only enhancement layer packets, especially the highly important base layer packets gain from the presence of the traffic management module. Although in the sce- 
narios where the traffic managment module is used it is observed that the base layer packets experience a higher packet loss probability at the bottleneck buffer and at the dejittering buffer than the enhancement layer packets, it appears that if the sum of $T_{j i t}+d^{*}$, of the dejittering delay $T_{j i t}$ and of the queueing delay experienced at the bottleneck buffer by the first packet of the video stream $d^{*}$, is larger than the threshold $T h$ used in the scenario, then a base layer packet has a higher probability of reaching the receiver than an enhancement layer packet.

So the choice of the position of the threshold in the bottleneck buffer and of the dejittering delay at the dejittering buffer cannot be uncoupled. A general guideline is that $T h$ should always be chosen low enough such that the packet loss of the base layer packets at the bottleneck buffer is below a required target, but preferable somewhere in the higher part of that buffer because otherwise there will be a large part of the enhancement layer packets that will never reach their receiver because they are unnecessarily dropped by the traffic management module. Once the threshold position is chosen, the dejittering delay $T_{j i t}$ should be chosen such that $T_{j i t}+d^{*}$ is larger than $T h$, because then the packet loss due to dejittering buffer underflow lies in the region where the underflow probability goes down more steeply with increasing $T_{j i t}+d^{*}$.

Remark that with the network scenario considered in this paper, where the traffic management module is present immediately before the bottleneck buffer, the packets that are discarded by the traffic management module first travel (unnecessary) from the video source through the core and aggregation network until the access network. Another option would be to make the video sources responsive, as with DCCP, such that the sources themselves discard the enhancement layer packets in case of congestion, based on feedback signals they receive from the receiver about the congestion status of the network. If this feedback arrives with a negligible delay, the model and the results presented in this paper stay valid. When the feedback signals arrive however at the sources with a non-negligible delay, the results will change. The construction of a model that can assess the performance of such a network scenario, and a comparison of its performance with that of the network scenario considered in the current paper, will be the topic of some future work.

\section{References}

1. Sharpe, R., Zriny, D., De Vleeschauwer, D.: Access network enhancements for the delivery of video services. Alcatel Telecom Review (ATR), pp. 134-140 (2005)

2. Schwarz, H., Marpe, D., Wiegand, T.: Overview of the scalable H.264/MPEG4AVC extension. In: Proceedings of the IEEE International Conference on Image Processing (ICIP'06) (2006)

3. Kohler, E., Handley, M., Floyd, S.: Datagram congestion control protocol (DCCP). RFC 4340 (2006)

4. Anderson, N.: An introduction to IPTV (2006), http://arstechnica.com/ guides/other/iptv.ars/

5. Perkins, C.: RTP - Audio and Video for the Internet. Addison-Wesley, New York (2003) 